\title{
An NRAS mutation in primary malignant melanoma of the lung: a case report
}

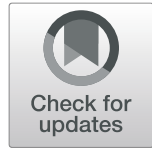

\author{
Takashi Hibiya ${ }^{1,2^{*}}$ (D, Meiro Tanaka ${ }^{3}$, Mai Matsumura ${ }^{3}$, Ayako Aoki ${ }^{4}$, Tadashi Ikegami ${ }^{5,6}$, Koji Okudela $^{3}$, \\ Naomi Kawano ${ }^{2}$ and Kenichi Ohashi ${ }^{1,3}$
}

\begin{abstract}
Background: Primary malignant melanoma of the lung (PML) is extremely rare. No precursor lesions of PML have been identified, and little is known about the genetic mutations associated with the disease. Typically, 15-20\% of malignant melanomas possess NRAS gene mutations, but no cases of NRAS-mutated PML have been reported in the English literature. We present a case of PML involving an NRAS mutation.
\end{abstract}

Case presentation: Clinical summary

A 74-year-old Japanese female presented with worsening dyspnea and was admitted to hospital. Computed tomography (CT) revealed a right lung (S10) mass and pleural dissemination. Cytology of the pleural effusion in the right lung was performed, and malignant melanoma or clear cell sarcoma was suspected. A dermatological examination and gallium scintigraphy were conducted to determine the primary tumor site, but no suspicious lesions, expect for the right lung mass, were found. After admission, CT showed complicating bilateral pneumonia, and an antibiotic drug was administered, but the pleural effusion got worse. About 2 weeks later, the patient died of respiratory failure and cardiac arrest. An autopsy was performed to determine the histological diagnosis. Autopsy findings A 26x15×20-mm black and pale yellow mass was found in the right lower lobe. Many disseminated nodules were found in the right lobe. The tumor had invaded the right diaphragm. Subcarinal lymph node metastasis was also detected. Immunohistochemically, the tumor cells exhibited positivity for S-100 and HMB45 staining. The patient was diagnosed with malignant melanoma. Sanger sequencing of the tumor detected an NRAS mutation.

Conclusions: We found an NRAS D54N mutation in PML, which has not been reported previously anywhere in the world. Previous reports indicated that most cases of PML can be classified into the triple-wild-type, but BRAF mutation status was only analyzed in a few cases. We should analyze the mutation patterns of PML to determine whether any subtypes other than the triple-wild-type exist. PML might be a form of de novo cancer.

Keywords: Primary malignant melanoma of the lung, NRAS mutation, Sanger sequencing, Autopsy

\section{Background}

Primary malignant melanoma of the lung is extremely rare and only accounts for $0.01 \%$ of all primary lung tumors [1]. The median age of patients with the condition is between 51 and 59 years, and the disease exhibits an approximately equal sex distribution or a slight male predominance [2]. No precursor lesions of primary malignant melanoma of

\footnotetext{
* Correspondence: hibiya-cib@umin.ac.jp

'Department of Pathology, Yokohama City University Hospital, 3-9 Fukuura, Kanazawa-ku, Yokohama 236-0004, Japan

${ }^{2}$ Department of Pathology, Yokohama Minami Kyousai Hospital, 1-21-1

Mutsuura-higashi, Kanazawa-ku, Yokohama 236-0037, Japan

Full list of author information is available at the end of the article
}

the lung have been identified [2], and the associated genetic mutations are poorly understood. In one case, it was reported that pulmonary malignant melanoma carried a tumor protein p53 (TP53) mutation [3]. Typically, 15-20\% of malignant melanomas have mutations in the neuroblastoma RAS viral oncogene homolog (NRAS) gene [4], but no cases of NRAS-mutated primary malignant melanoma of the lung have been reported in the English literature. We present the case of a 74-year-old female, who died of primary malignant melanoma of the lung involving an NRAS mutation.

(c) The Author(s). 2020 Open Access This article is distributed under the terms of the Creative Commons Attribution 4.0 International License (http://creativecommons.org/licenses/by/4.0/), which permits unrestricted use, distribution, and reproduction in any medium, provided you give appropriate credit to the original author(s) and the source, provide a link to the Creative Commons license, and indicate if changes were made. The Creative Commons Public Domain Dedication waiver (http://creativecommons.org/publicdomain/zero/1.0/) applies to the data made available in this article, unless otherwise stated. 


\section{Case presentation}

\section{Clinical summary}

A 74-year-old Japanese female presented with worsening dyspnea and was admitted to hospital. A chest X-ray revealed right-sided pleural effusion and cardiac enlargement. Computed tomography (CT) showed a right lung (S10) mass and pleural dissemination (Fig. 1). Cytology of the pleural effusion in the right lung was performed, and tumor cells were obtained. The tumor cells had round nuclei, large and distinct nucleoli, and melanin particles in their cytoplasm. We suspected malignant melanoma or clear cell sarcoma (Fig. 2). A dermatological examination and gallium scintigraphy were conduced to determine the primary tumor site, but no suspicious lesions, expect the right lung mass, were found (Fig. 3).

After admission, CT showed ground-glass opacities in both lungs, and the patient was diagnosed with complicating bilateral pneumonia and was given an antibiotic drug. It was transiently effective, but the right pleural effusion got worse. About 2 weeks later, the patient died of respiratory failure and cardiac arrest. An autopsy was performed to determine the histological diagnosis.

\section{Autopsy findings}

A 26x15x20-mm black and pale yellow mass was found in the right lower lobe. Many disseminated nodules were found in the right lobe (Fig. 4). The tumor had invaded the right diaphragm. A subcarinal lymph node metastasis $(45 \times 21 \times 15 \mathrm{~mm}$ in size) was also detected. Pale bloodcolored, massive right-sided pleural effusion $(1850 \mathrm{ml})$ was noted, which was indicative of pleuritis carcinomatosa.

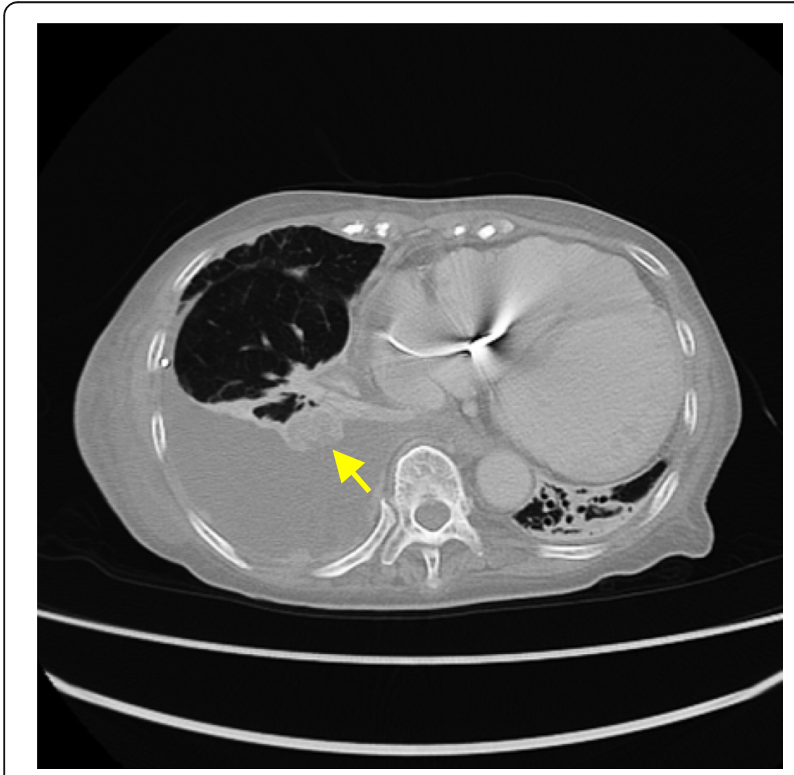

Fig. 1 Chest $C T$ indicated the presence of a right lung (S10) mass (arrow) and pleural dissemination

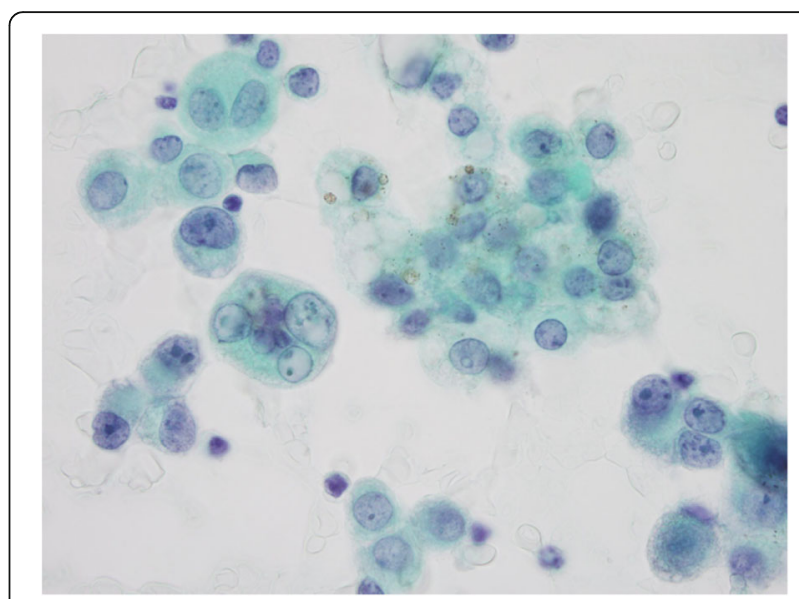

Fig. 2 Cytology of the right pleural effusion showed malignant cells, which led us to suspect malignant melanoma or clear cell sarcoma

Histologically, the tumor cells had large nuclei with high nuclear/cytoplasmic ratios, large and distinct nucleoli, and melanin particles in their cytoplasm. The tumor exhibited intraepithelial spread into a bronchus (Fig. 5). No primary tumor was found, expect in the right lower lobe. We

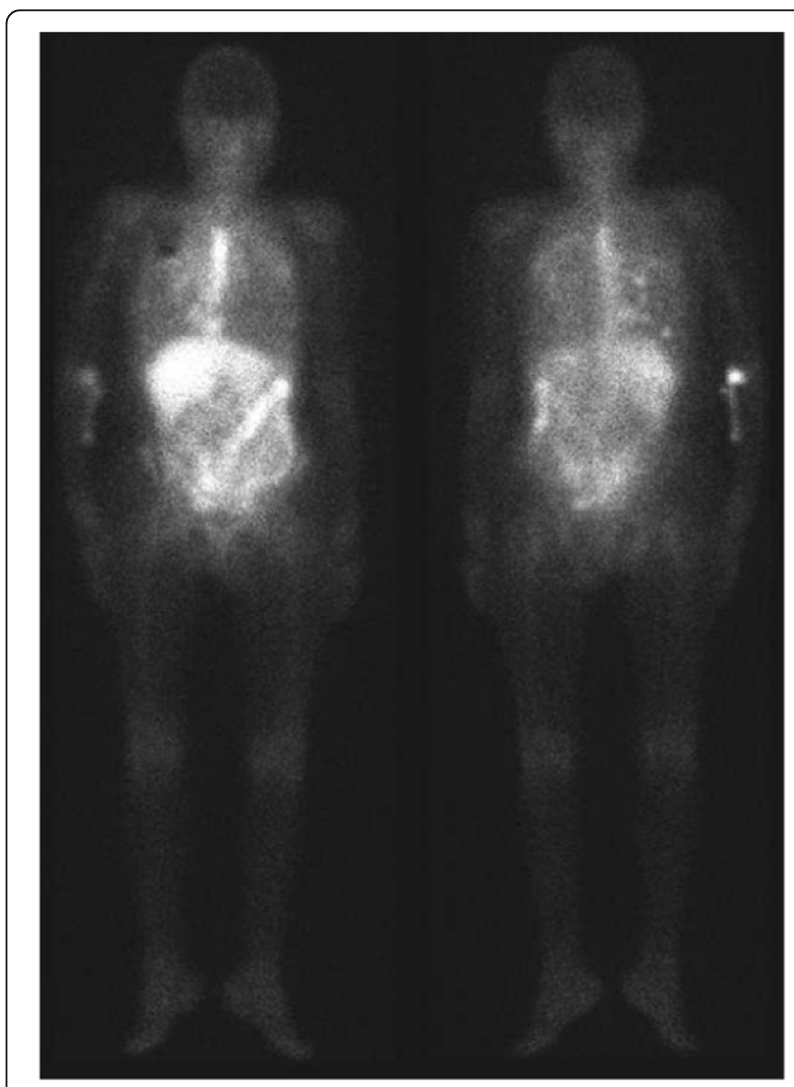

Fig. 3 Gallium scintigraphy did not reveal any suspicious lesions expect the right lung mass 


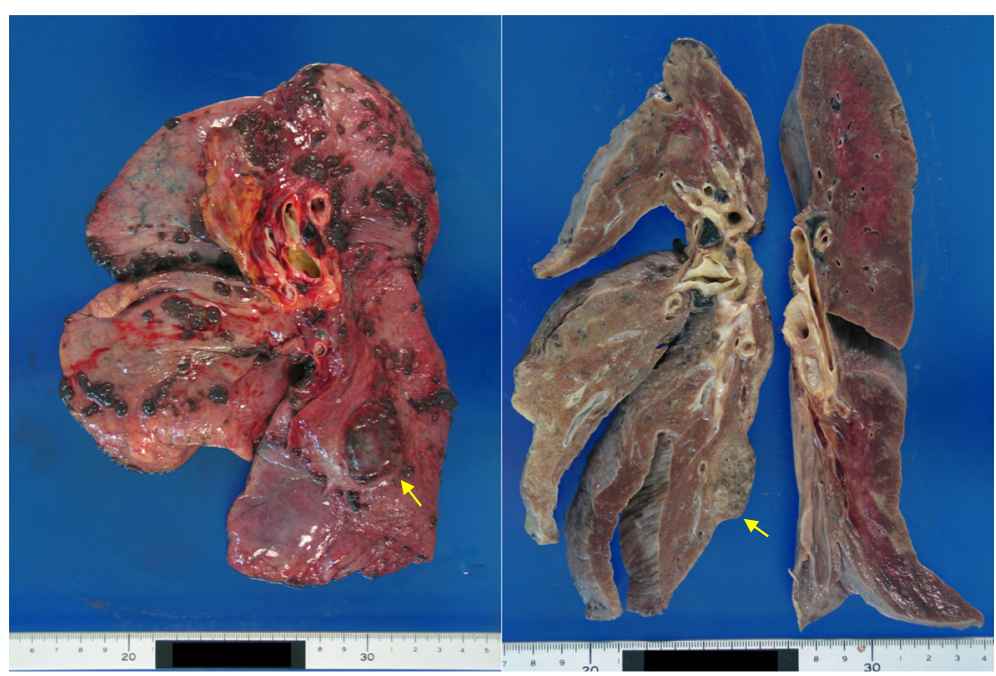

Fig. 4 A 26×15×20-mm black and pale yellow mass was found in the right lower lobe. Many disseminated nodules were found in the right lobe

performed immunohistochemical staining using an HMB45 antibody and antibodies against S-100 and c-kit. The tumor cells exhibited positivity for S-100 and HMB45 staining (Fig. 5), but were negative for c-kit. The patient was diagnosed with malignant melanoma.

We determined the tumor's proto-oncogene B-Raf $(B R A F)$ and NRAS mutational status using Sanger sequencing. Primers were designed to amplify $B R A F$ exon
15, NRAS exon 2, and NRAS exon 3 (Table 1). As a result, we detected an NRAS mutation (D54N) (Fig. 6). We also determined the KIT mutational status using Sanger sequencing. We sequenced KIT exon 8, 9, 11, 13, 17 and 18, but no KIT gene mutation was detected.

Apart from malignant melanoma, foamy macrophages exuded into the alveolar spaces of the bilateral lobes, and sputum had plugged the bronchi. Neutrophils had

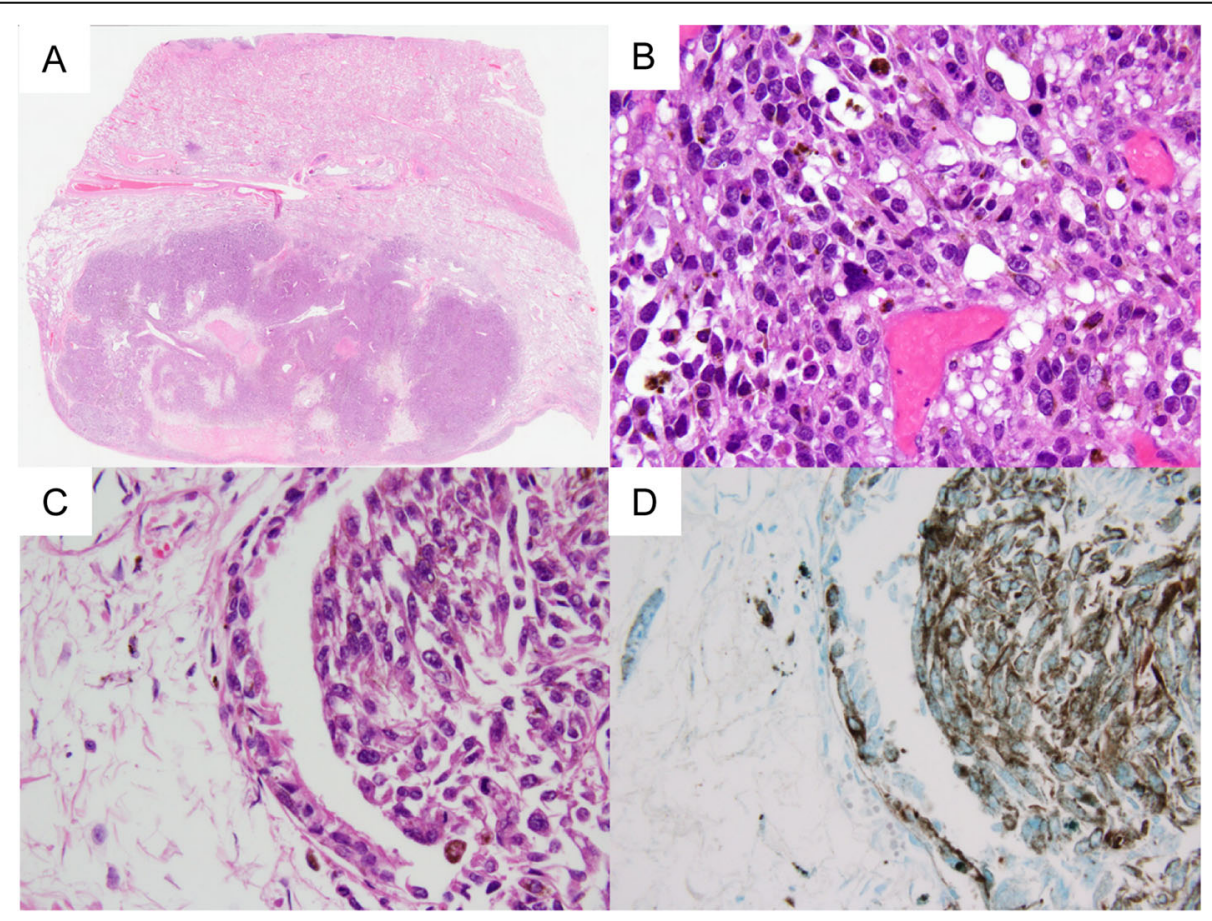

Fig. $\mathbf{5}$ a and $\mathbf{b}$ : Malignant melanoma had invaded the right lower lobe. (Hematoxylin and eosin [HE] staining). $\mathbf{c}$ and $\mathbf{d}$ : The melanoma exhibited intraepithelial spread into a bronchus. (c: HE staining, d: HMB45 staining). 
Table 1 Primers used for the Sanger sequencing

\begin{tabular}{lllcc}
\hline Gene & Exons & 5' $\rightarrow$ 3' Sequence & Tm & Length \\
\hline BRAF & EX15 & F: TCATAATGCTTGCTCTGATAGG & 60 & 224 \\
& & R: GGCCAAAAATTAATCAGTGG & & \\
NRAS & EX2 & F: GAAAGCTTAAAGTACTGTAGATGTGG & 60 & 247 \\
& & R: AGATGATCCGACAAGTGAGAGA & & \\
NRAS & EX3 & F: CCCCTTACCCTCCACACC & 60 & 243 \\
& & R: CACAAAGATCATCCTITCAGAGAA & & \\
& &
\end{tabular}

infiltrated into some alveoli and bronchi. We diagnosed the patient with lipoid pneumonia.

\section{Discussion}

Primary malignant melanoma of the lung is extremely rare, and little is known about the genetic mutations associated with the condition. A previous study showed that malignant melanoma exhibited a high prevalence of somatic mutations [5]. However, somatic mutation patterns vary substantially between melanoma subtypes [6]. Sun-exposed skin melanomas have the highest numbers of mutations. Many have neurofibromatosis type I (NF1) and NRAS mutations, and sporadic BRAF V600K mutations are also seen [4, 7]. Moderately sun-exposed skin melanomas (excluding chronic sun-damaged skin melanomas) have intermediate numbers of mutations. Some possess the BRAF V600E mutation, whereas NRAS mutations are rare $[4,8]$. Non-cutaneous melanomas have significantly lower numbers of mutations [4]. Ultraviolet (UV) light is cited as a possible major driver of mutagenesis in melanoma. As primary malignant melanoma of the lung is considered to be a non-UV related melanoma, it might exhibit few somatic mutations.

Previously, 73 cases of primary malignant melanoma of the lung have been reported in the English literature. However, mutation status was only analyzed in 9 cases (Table 2) [3, 9-15]. Of these 9 cases, all 9 were analyzed for $B R A F$ mutations, but only 3 cases were

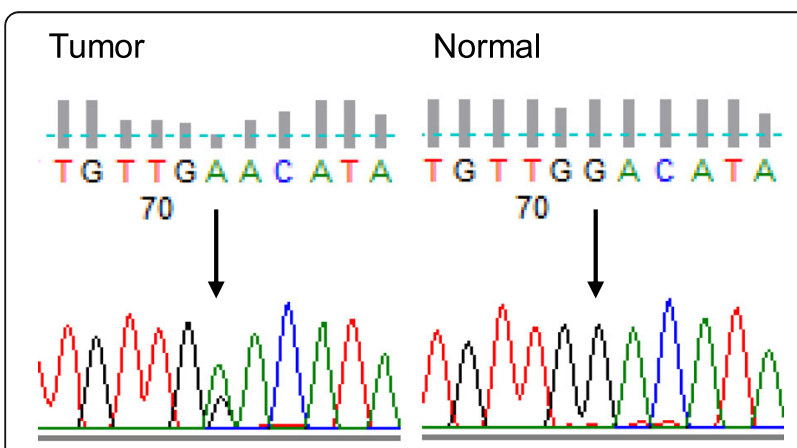

Fig. 6 The results of NRAS exon 3 Sanger sequencing of the tumor and normal tissue are shown. The tumor carried a mutation analyzed for NRAS and KIT mutations, and only 2 cases were analyzed for TP53 mutations. A TP53 P72R mutation was detected in one case [3], but no BRAF or $N R A S$ mutations were found. We detected an NRAS D54N mutation in our case, which is the first time an NRAS mutation has been detected in primary malignant melanoma of the lung anywhere in the world.

Information about the genomic classification of malignant melanoma was previously reported in a classification of cutaneous melanoma [16]. According to the report, cutaneous melanoma can be classified into four subtypes: mutant BRAF; mutant NRAS; mutant NF1; and the triplewild-type, which is characterized by a lack of hot-spot $B R A F, N / H / K R A S$, or NF1 mutations. This genomic classification might aid the selection of therapeutic targets [4]. Almost all primary malignant melanomas of the lung are classified into the triple-wild-type, and few are classified into the mutant NRAS type. However, the mutation status of primary malignant melanoma of the lung has not been sufficiently analyzed yet. So, when we encounter malignant melanoma of the lung, we should analyze not only $B R A F$ mutations, but also other mutations.

In our case, we detected an NRAS D54N mutation. Most of the RAS gene mutations found in cancer are missense mutations, with $98 \%$ of these mutations being located at the G12, G13, or Q61 hotspot [17]. D54N is an extremely rare mutation. In the Catalogue Of Somatic Mutations In Cancer (COSMIC) v88, the NRAS D54N mutation was only reported in one case of colon adenocarcinoma. The function of D54N-mutated NRAS is not yet known.

No precursor lesions of primary malignant melanoma of the lung have yet been identified [2]. Some of the authors previously reported a case of pulmonary melanocytic nevus. The nevus cells of the pulmonary melanocytic nevus exhibited a BRAF V600E mutation, but no NRAS mutations were found [18]. No BRAF mutations were detected in the 9 previously reported cases of primary malignant melanoma of the lung or our case; therefore, primary malignant melanoma of the lung might be a form of de novo cancer, rather than arise from melanocytic nevus.

\section{Conclusions}

We found an NRAS D54N mutation in primary malignant melanoma of the lung, which has not been reported previously anywhere in the world. Previously reported cases have demonstrated that non-UV related melanomas have lower numbers of mutations than cutaneous melanoma, and most cases of primary malignant melanoma of the lung can be classified into the triple-wildtype because they are non-UV related tumors. However, the $B R A F$ mutation status of primary malignant melanoma of the lung has only been analyzed in 9 previous 
Table 2 Nine cases of primary malignant melanoma of the lung in which mutation status was analyzed

\begin{tabular}{|c|c|c|c|c|c|c|c|c|}
\hline & & & & & Mutation & & & \\
\hline No. & Author & Year & Age & Sex & BRAF & NRAS & KIT & TP53 \\
\hline 1 & dos Santos et al. & 2013 & 62 & $\mathrm{~F}$ & Negative & N/A & N/A & N/A \\
\hline 2 & Watanabe et al. & 2015 & 66 & M & Negative & Negative & Negative & P72R \\
\hline 3 & & & 46 & $\mathrm{~F}$ & Negative & Negative & Negative & Negative \\
\hline 4 & Hirai et al. & 2017 & 86 & $\mathrm{~F}$ & Negative & N/A & N/A & N/A \\
\hline 5 & Kyriakopoulos et al. & 2017 & 56 & $\mathrm{~F}$ & Negative & Negative & Negative & N/A \\
\hline 6 & Yamamoto et al. & 2017 & 61 & $\mathrm{~F}$ & Negative & N/A & N/A & N/A \\
\hline 7 & Holmes and Chung & 2017 & 43 & $\mathrm{~F}$ & Negative & N/A & N/A & N/A \\
\hline 8 & Shi et al. & 2018 & 46 & M & Negative & N/A & N/A & N/A \\
\hline 9 & Yabuki et al. & 2018 & 74 & M & Negative & N/A & N/A & N/A \\
\hline 10 & Our case & & 74 & $\mathrm{~F}$ & Negative & D54N & Negative & N/A \\
\hline
\end{tabular}

Abbreviations: N/A indicates not available

cases, and other types of mutations have only been analyzed in 3 previous cases. We should analyze mutation patterns to determine whether some primary malignant melanomas of the lung belong to mutation subtypes other than the triple-wild-type.

Primary malignant melanoma of the lung might be a form of de novo cancer.

\section{Abbreviations}

COSMIC: Catalogue Of Somatic Mutations In Cancer; CT: Computed tomography; HE: Hematoxylin and eosin; NF1: Neurofibromatosis type l; NRAS: Neuroblastoma RAS viral oncogene homolog; PML: Primary malignant melanoma of the lung; TP53: Tumor protein p53; UV: Ultraviolet

\section{Acknowledgements}

Not applicable

\section{Authors' contributions}

TH performed the autopsy, researched the patient's background, and drafted the manuscript. MT and MM carried out the DNA sequencing. AA provided critical information and insights about pneumological aspects of this case. TI provided critical information and insights about radiological aspects of this case. NK provided insights into pathological aspects of this case. KOK provided insights into the pathological aspects of this case and corrected the first version of the draft. KOh critically revised the manuscript. All of the authors have approved the final version of the text.

\section{Funding}

The authors have no funding to disclose.

\section{Availability of data and materials}

Not applicable

\section{Ethics approval and consent to participate}

This study was approved by the ethics committee of the Yokohama Minami Kyousai Hospital.

\section{Consent for publication}

We obtained informed consent from the patient's family for the publication of this case report.

\section{Competing interests}

The authors declare that they have no competing interests.

\section{Author details}

1Department of Pathology, Yokohama City University Hospital, 3-9 Fukuura, Kanazawa-ku, Yokohama 236-0004, Japan. ²Department of Pathology, Yokohama Minami Kyousai Hospital, 1-21-1 Mutsuura-higashi, Kanazawa-ku, Yokohama 236-0037, Japan. ${ }^{3}$ Department of Pathology, Graduate School of Medicine, Yokohama City University, 3-9 Fukuura, Kanazawa-ku, Yokohama 236-0004, Japan. ${ }^{4}$ Department of Respiratory Medicine, Yokohama Minami Kyousai Hospital, 1-21-1 Mutsuura-higashi, Kanazawa-ku, Yokohama 236-0037, Japan. ${ }^{5}$ Department of Diagnostic Radiology, Kanagawa Dental University Hospital, 1-23 Ogawacho, Yokosuka 238-8570, Japan. ${ }^{6}$ Department of Radiology, Yokohama Minami Kyousai Hospital, 1-21-1 Mutsuura-higashi, Kanazawa-ku, Yokohama 236-0037, Japan.

Received: 8 November 2019 Accepted: 28 January 2020

Published online: 07 February 2020

\section{References}

1. Wilson RW, Moran CA. Primary melanoma of the lung: a clinicopathologic and immunohistochemical study of eight cases. Am J Surg Pathol. 1997; 21(10):1196-202.

2. Travis WD, Brambilla E, Burke A, Marx A, Nicholson AG, International Agency for Research on Cancer. WHO classification of tumours of the lung, pleura, thymus and heart. Lyon. 2015:146.

3. Watanabe M, Yamamoto H, Hashida S, Soh J, Sugimoto S, Toyooka S, et al. Primary pulmonary melanoma: a report of two cases. World J Surg Oncol. 2015;13:274

4. Davis EJ, Johnson DB, Sosman JA, Chandra S. Melanoma: what do all the mutations mean? Cancer. 2018:124(17):3490-9.

5. Alexandrov LB, Nik-Zainal S, Wedge DC, Aparicio SA, Behjati S, Biankin AV, et al. Signatures of mutational processes in human cancer. Nature. 2013; 500(7463):415-21.

6. Hayward NK, Wilmott JS, Waddell N, Johansson PA, Field MA, Nones K, et al. Whole-genome landscapes of major melanoma subtypes. Nature. 2017; 545(7653):175-80.

7. Krauthammer M, Kong Y, Ha BH, Evans P, Bacchiocchi A, McCusker JP, et al. Exome sequencing identifies recurrent somatic RAC1 mutations in melanoma. Nat Genet. 2012:44(9):1006-14.

8. Maldonado JL, Fridlyand J, Patel H, Jain AN, Busam K, Kageshita T, et al. Determinants of BRAF mutations in primary melanomas. J Natl Cancer Inst. 2003;95(24):1878-90.

9. dos Santos CL, Fernandes LR, Meruje M, Barata F. Primary pulmonary melanoma: the unexpected tumour. BMJ Case Rep. 2013;2013: bcr2013200706

10. Hirai I, Tanese K, Obata S, Funakoshi T. A case of primary malignant melanoma of the lung responded to anti-PD-1 antibody therapy. Indian J Thorac Cardiovasc Surg. 2017;33(2):173-5. 
11. Kyriakopoulos C, Zarkavelis G, Andrianopoulou A, Papoudou-Bai A, Stefanou D, Boussios $S$, et al. Primary pulmonary malignant melanoma: report of an important entity and literature review. Case Rep Oncol Med. 2017:2017:8654326.

12. Yamamoto Y, Kodama K, Maniwa T, Takeda M, Tanaka Y, Ozawa K, et al. Primary malignant melanoma of the lung: a case report. Mol Clin Oncol. 2017;7(1):39-41

13. Holmes A, Chung J. Two Cases of Primary Extracutaneous Melanoma: Primary Gastric Melanoma and Primary Melanoma of the Lung. Med Case Rep. 2017;3(4:39). https://doi.org/10.21767/2471-8041.100069.

14. Shi Y, Bing Z, Xu X, Cui Y. Primary pulmonary malignant melanoma: case report and literature review. Thorac Cancer. 2018;9(9):1185-9.

15. Yabuki H, Kuwana K, Minowa M. Resection of primary malignant lung melanoma: a case report. Asian Cardiovasc Thorac Ann. 2018;26(9):710-2.

16. Network CGA. Genomic classification of cutaneous melanoma. Cell. 2015; 161(7):1681-96.

17. Hobbs GA, Der CJ, Rossman KL. RAS isoforms and mutations in cancer at a glance. J Cell Sci. 2016;129(7):1287-92.

18. Tanaka M, Matsumura M, Okudela K, Mitsui H, Tateishi Y, Umeda S, et al. Pulmonary melanocytic nevus - a case report with a mutation analysis of common driver oncogenes. Pathol Int. 2019;69(11):667-71. https://doi.org/ 10.1111/pin.12850

\section{Publisher's Note}

Springer Nature remains neutral with regard to jurisdictional claims in published maps and institutional affiliations.

Ready to submit your research? Choose BMC and benefit from:

- fast, convenient online submission

- thorough peer review by experienced researchers in your field

- rapid publication on acceptance

- support for research data, including large and complex data types

- gold Open Access which fosters wider collaboration and increased citations

- maximum visibility for your research: over $100 \mathrm{M}$ website views per year

At BMC, research is always in progress.

Learn more biomedcentral.com/submissions 